\title{
Discriminatory effects in the optical binding of chiral nanoparticles
}

ARTICLE in PROC SPIE · AUGUST 2015

DOI: $10.1117 / 12.2188147$

3 AUTHORS:

Kayn A. Forbes

University of East Anglia

5 PUBLICATIONS 4 CITATIONS

SEE PROFILE

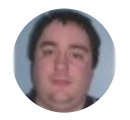

David S Bradshaw

University of East Anglia

73 PUBLICATIONS 477 CITATIONS

SEE PROFILE

David L Andrews

University of East Anglia

347 PUBLICATIONS 3,003 CITATIONS

SEE PROFILE 


\title{
Discriminatory effects in the optical binding of chiral nanoparticles
}

\author{
Kayn A. Forbes, David S. Bradshaw, and David L. Andrews* \\ School of Chemistry, University of East Anglia, Norwich Research Park, Norwich NR4 7TJ, U.K.
}

\begin{abstract}
The laser-induced intermolecular force that exists between two or more particles subjected to a moderately intense laser beam is termed 'optical binding'. Completely distinct from the single-particle forces that give rise to optical trapping, the phenomenon of optical binding is a manifestation of the coupling between optically induced dipole moments in neutral particles. In conjunction with optical trapping, the optomechanical forces in optical binding afford means for the manipulation and fabrication of optically bound matter. The Casimir-Polder potential that is intrinsic to all matter can be overridden by the optical binding force in cases where the laser beam is of sufficient intensity. Chiral discrimination can arise when the laser input has a circular polarization, if the particles are themselves chiral. Then, it emerges that the interaction between particles with a particular handedness is responsive to the left- or right-handedness of the light. The present analysis, which expands upon previous studies of chiral discrimination in optical binding, identifies a novel mechanism that others have previously overlooked, signifying that the discriminatory effect is much more prominent than originally thought. The new theory leads to results for freely-tumbling chiral particles subjected to circularly polarized light. Rigorous conditions are established for the energy shifts to be non-zero and display discriminatory effects with respect to the handedness of the incident beam. Detailed calculations indicate that the energy shift is larger than those previously reported by three orders of magnitude.
\end{abstract}

Keywords: optical forces, optical binding, optically bound matter, quantum electrodynamics, circularly polarized light, chirality, chiral discrimination, nanoparticles

\section{INTRODUCTION}

Optical binding is an optomechanical phenomenon, predicted by Thirunamachandran ${ }^{1}$ and distinct from optical trapping, where the applied electromagnetic radiation not only traps but also induces an optical force between particles. These laser-induced forces can be either attractive or repulsive (despite an implication of the former in the usually applied term "binding") and it may override the attractive inter-particle Casimir-Polder force ${ }^{2}$ that always exists. The earliest experimental efforts focused on binding between dielectric microparticles ${ }^{3-8}$ but increasing attention has more recently been given to nanoparticle systems. ${ }^{9-11}$ Theoretical developments on the latter (for example see refs 12,13 and references therein) include molecular studies based on quantum theory. ${ }^{14-16}$ Here, our attention is drawn to the novel effects that arise when both the trapped nanoparticles and input light are chiral, with either the same or the opposite sense of handedness. Optical forces are said to be discriminatory when different physical results arise for opposite handedness of molecules and/or the applied circularly polarized beam. These discriminatory effects may be attributed to low molecular symmetry, such that selection rules allow at least some electronic transitions to include contributions from not only electric dipole, but also other electric and magnetic multipole moments. Examples of discriminatory optical processes include circular dichroism ${ }^{17}$ and circular differential scattering ${ }^{18}$ whilst recent investigations have also revealed that discriminatory optical forces can occur in optical trapping. ${ }^{19}$ Although small in comparison to forces mediated by electric-electric dipole interactions, the leading chiral-dependent term typically corresponds to electric-magnetic dipole couplings. Some chiral aspects of optical binding have been identified previously; ${ }^{20}$ this earlier study showed that the discriminatory effects are of the order of $10^{-6}$ smaller than the electric-electric dipole coupling forces, and independent of the handedness of incident radiation. In a more recent analysis ${ }^{21}$ it has been revealed that the electric-magnetic terms that contribute, which were not considered in the previous work, are in fact of high physical importance - meaning that the chiral binding force is typically several orders of magnitude greater than previously thought and, moreover, dependent on both the handedness of the radiation and the trapped particles.

Optical Trapping and Optical Micromanipulation XII, edited by Kishan Dholakia, Gabriel C. Spalding Proc. of Vol. 9548, 95480M - (c) 2015 SPIE · CCC code: 0277-786X/15/\$18 · doi: 10.1117/12.2188147 


\section{SUMMARY OF THE DERIVATION}

In the following derivation the method of induced moments ${ }^{22-24}$ is used to calculate the laser-induced shift in energy created between a pair of chiral particles in the presence of a circularly polarized beam. The physical basis of the method of induced moments is that electromagnetic fields induce electric multipole $\left(\mathrm{E}_{n}\right)$ and magnetic multipole $\left(\mathrm{M}_{n}\right)$ moments in polarizable bodies, and as a consequence multipole-multipole coupling occurs via resonant interaction. The leading contribution to optical binding forces comes from a coupling between the polarizabilities of the two particles $\left(E 1^{2}-E 1^{2}\right.$ coupling) and it is non-discriminatory with regard to chirality. The original studies ${ }^{25}$ on the discriminatory forces at play in optical binding were concerned with a polarizability variant (E1M1-E1M1) coupling mechanism between chiral particles. Although $\mathrm{E} 1^{2}$ couplings are non-discriminatory, it is possible for one chiral particle to engage its $E 1^{2}$ properties with the E1M1 properties of another chiral particle to produce E1 ${ }^{2}$-E1M1 couplings - which, owing to the E1M1 component are discriminatory. The E1 ${ }^{2}$-E1M1 energy shift for a pair of chiral molecules is represented as;

$$
\begin{aligned}
\Delta E= & {\left[\mu_{i}^{\operatorname{ind}(\alpha)}(A) \mu_{j}^{\operatorname{ind}(G)}(B)+\mu_{i}^{\operatorname{ind}(G)}(A) \mu_{j}^{\operatorname{ind}(\alpha)}(B)\right] \operatorname{Re} V_{i j}(k, \mathbf{R}) } \\
& +\left[\mu_{i}^{\operatorname{ind}(\alpha)}(A) m_{j}^{\operatorname{ind}(G)}(B)+m_{i}^{\operatorname{ind}(G)}(A) \mu_{j}^{\operatorname{ind}(\alpha)}(B)\right] \operatorname{Im} U_{i j}(k, \mathbf{R}),
\end{aligned}
$$

where the induced moments $\mu_{i}^{\operatorname{ind}(\alpha)}, \mu_{i}^{\operatorname{ind}(G)}$, and $m_{j}^{\operatorname{ind}(G)}$ are given by,

$$
\begin{aligned}
& \mu_{i}^{\operatorname{ind}(G)}(\xi)=G_{i j}(\xi, \omega) b_{j}\left(\mathbf{R}_{\xi}\right), \\
& \mu_{i}^{\operatorname{ind}(\alpha)}(\xi)=\varepsilon_{0}^{-1} \alpha_{i j}(\xi, \omega) d_{j}^{\perp}\left(\mathbf{R}_{\xi}\right), \\
& m_{j}^{\operatorname{ind}(G)}(\xi)=-\varepsilon_{0}^{-1} G_{j i}^{\prime}(\xi, \omega) d_{i}^{\perp}\left(\mathbf{R}_{\xi}\right) .
\end{aligned}
$$

Here, the non-discriminatory $\mathrm{E} 1^{2}$ interaction is represented by the electric-dipole polarizability $\alpha_{i j}(\xi, \omega)$ of a species $\xi$ and the discriminatory mixed electric-magnetic counterpart, E1M1, is denoted by $G_{i j}(\xi, \omega)$, which changes sign when one enantiomer is replaced by another of opposite handedness. The explicit forms of $\alpha_{i j}(\xi, \omega)$ and $G_{i j}(\xi, \omega)$ are given in ref. 21. It is noteworthy that whilst $\alpha_{i j}(\xi, \omega)$ is a real quantity, $G_{i j}(\xi, \omega)$ is imaginary. Here, $d_{i}^{\perp}\left(\mathbf{R}_{\xi}\right)$ and $b_{j}\left(\mathbf{R}_{\xi}\right)$ are the transverse displacement electric field operator and magnetic field operators, respectively. In equation $(1), V_{i j}(k, \mathbf{R})$ and $U_{i j}(k, \mathbf{R})$ represent retarded resonant dipole-dipole interaction tensors, for E1-E1 and E1-M1 couplings, respectively. ${ }^{26}$ Expanding the induced moments in (1) we find;

$$
\begin{aligned}
\Delta E=\varepsilon_{0}^{-1} & {\left[\alpha_{i k}(A, \omega) G_{l j}^{\prime}(B, \omega) d_{k}^{\perp}\left(\mathbf{R}_{A}\right) b_{l}\left(\mathbf{R}_{B}\right)+G_{k i}(A, \omega) \alpha_{l j}(B, \omega) b_{k}\left(\mathbf{R}_{A}\right) d_{l}^{\perp}\left(\mathbf{R}_{B}\right)\right] } \\
& \times \operatorname{Re} V_{i j}(k, \mathbf{R})+\varepsilon_{0}^{-2}\left[\alpha_{i k}(A, \omega) G_{j l}(B, \omega)+G_{i k}^{\prime}(A, \omega) \alpha_{l j}(B, \omega)\right] \\
& \times d_{k}^{\perp}\left(\mathbf{R}_{A}\right) d_{l}^{\perp}\left(\mathbf{R}_{B}\right) \operatorname{Im} U_{i j}(k, \mathbf{R}),
\end{aligned}
$$

where, due to the time-odd character of the magnetic-dipole operator (and with the magnetic transition dipoles satisfying the relation $-m_{i}^{r 0}=m_{i}^{0 r}$ ), we use the identity $-G_{i j}=G_{j i}^{\prime}$. Thus, equation (5) can be understood as the coupling of the polarizability tensor, $\alpha$, of one particle with the $G$ tensor of the other, which we can write in shorthand as " $\alpha-G$ " coupling. In addition, $\mathrm{E} 1^{2}$-E $1^{2}$ involves " $\alpha-\alpha$ " coupling and E1M1-E1M1, "G-G" coupling. Further manipulation of equation (5) is possible by taking the expectation values of both the molecular and radiation parts, and carrying out an orientational average using standard techniques, ${ }^{27}$ i.e.: 


$$
\begin{aligned}
\Delta E=\frac{n \hbar k}{\varepsilon_{0} V}\{ & {\left[\alpha(A, \omega) G^{\prime}(B, \omega) e_{i}^{(\lambda)}(\mathbf{k}) \bar{b}_{j}^{(\lambda)}(\mathbf{k})+G(A, \omega) \alpha(B, \omega) b_{i}^{(\lambda)}(\mathbf{k}) \bar{e}_{j}^{(\lambda)}(\mathbf{k})\right] } \\
& \times \operatorname{Re} V_{i j}(k, \mathbf{R})+c\left[\alpha(A, \omega) G(B, \omega)+G^{\prime}(A, \omega) \alpha(B, \omega)\right] e_{i}^{(\lambda)}(\mathbf{k}) \bar{e}_{j}^{(\lambda)}(\mathbf{k}) \\
& \left.\times \operatorname{Im} U_{i j}(k, \mathbf{R})\right\} \cos (\mathbf{k} \cdot \mathbf{R}) .
\end{aligned}
$$

This expression represents an energy shift between two particles within a quantization volume, $V$.

\section{RESULTS AND ANALYSIS}

At this stage it becomes evident that the polarization state of the input light dictates the observed behavior. For a pair of particles in either of the $\mathbf{k} \| \mathbf{R}$ or $\mathbf{k} \perp \mathbf{R}$ configurations, the radiation-induced energy shift (6) is zero when the incident light is linearly polarized; however, the use of circularly polarized light produces non-zero energy shifts for both possible configurations, which are given below. Given a specific enantiomer, the superscript L/R on the left-hand side of each equation can be taken to signify the handedness of the radiation, correlating with the plus/minus signs on the right;

$$
\begin{gathered}
\Delta E^{\perp(\mathrm{L} / \mathrm{R})}=\frac{I}{8 \pi \varepsilon_{0}^{2} c^{2} R^{3}}\left\{\left(\tilde{G}(A, \omega) \alpha(B, \omega)-\alpha(A, \omega) \tilde{G}^{\prime}(B, \omega)\right)\right. \\
\left.\times\left[ \pm(\cos k R+k R \sin k R) \pm k^{2} R^{2} \cos k R\right]\right\}, \\
\Delta E^{\lceil(\mathrm{L} / \mathrm{R})}=\frac{I}{4 \pi \varepsilon_{0}^{2} c^{2} R^{3}}\left\{\left(\tilde{G}(A, \omega) \alpha(B, \omega)-\alpha(A, \omega) \tilde{G}^{\prime}(B, \omega)\right)\right. \\
\left.\times\left[\mp(\cos k R+k R \sin k R) \pm k^{2} R^{2} \cos k R\right]\right\} \cos (k R),
\end{gathered}
$$

where $I$ denotes the input laser irradiance (equal to $n \hbar c^{2} k / V$ ) and to further simplify notation we have used $\tilde{G}^{\prime}(\xi, \omega)=i G^{\prime}(\xi, \omega)$. Furthermore, we carry out a phased-weighted pair orientational average, ${ }^{28}$ which involves averaging over all $\mathbf{k}$ relative to $\mathbf{R}$ and over the relative orientations of the pair of molecules in the system, as is appropriate when the particle pair is in a liquid state. As with the previous results (7) and (8), the energy shift for a pair of freely tumbling chiral particles is zero if the incident radiation is linearly polarized. For circularly-polarized light the result is non-zero and the total phased-averaged energy shift is given as;

$$
\begin{aligned}
\left\langle\Delta E^{(L / R)}\right\rangle= \pm \frac{I}{4 \pi \varepsilon_{0}^{2} c^{2} R^{3}}( & \left.\alpha(A, \omega) \tilde{G}^{\prime}(B, \omega)-\tilde{G}(A, \omega) \alpha(B, \omega)\right) \\
& \times\left(\cos 2 k R-\frac{k R \sin 2 k R}{2}+\frac{3 \sin 2 k R}{2 k R}+\frac{3 \cos 2 k R}{(k R)^{2}}-\frac{3 \sin 2 k R}{2(k R)^{3}}\right) .
\end{aligned}
$$

As with the dominant electric dipole " $\alpha-\alpha$ " coupling, the result depends linearly on the irradiance of the input beam. The discriminatory effects embedded within the " $\alpha-G$ " coupling arise from both the handedness of the radiation and of the particles. Recalling that a particle's $G$ tensor is of opposite sign for either the right- or left-handed enantiomer, it is evident the discriminatory binding energy contributions (7), (8), and (9) are zero for a pair of opposite enantiomers, but non-zero for a pair of chiral particles of the same handedness. Moreover, the sign of the energy shift depends on both the particle and of the radiation handedness. For example, a pair of right-handed particles irradiated by right-handed light has the same discriminatory binding force as the corresponding pair of left-handed particles irradiated by lefthanded light. It is worth noting, though, that the energy shift would in fact be non-zero for a right- and left-handed pair of non-identical particles, since the magnitudes of their $G$ tensors would then differ. 
Due to the discriminatory nature of the non-zero binding force, where a pair of chiral particles are of the same handedness, any modulation of the light beam between right- and left-handed circular polarizations will correspondingly cause an oscillation in the equilibrium positions of the pair of chiral particles, as shown in Figure 1 by the dashed line. That is, since the equilibrium distance differs on comparing a pair of right-handed particles irradiated by right-handed light with the same pair irradiated with left-handed light. Tuning of the modulation frequency into resonance with the natural frequency of the optically bound pair should enhance the small-scale oscillations and allow them to become more readily detectable. Such a process would facilitate the identification and characterization of the chiral nature of the bound particles.

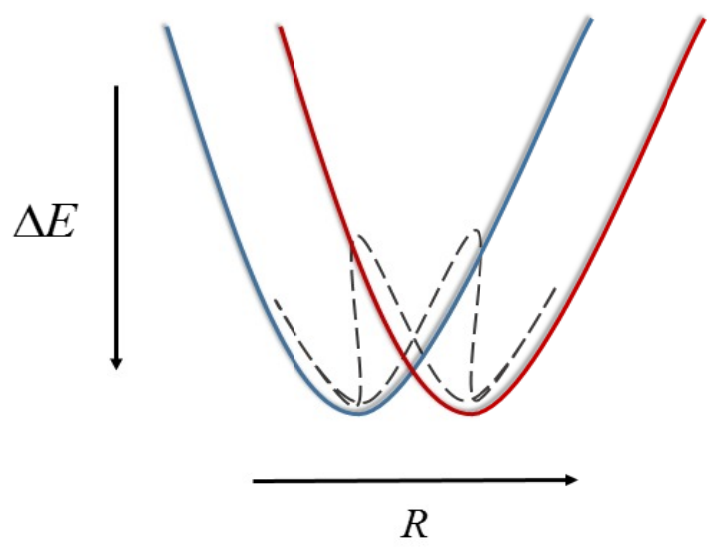

Figure 1 . The two energy minima for a pair of opposite enantiomers irradiated by circularly polarized light, where one curve corresponds to right-handed light and the other left-handed light. The dashed line represents the variation of the inter-particle separation distance through resonant modulation of the light.

\section{CONCLUSION}

The aim of this work has been to demonstrate that there are discriminatory optical binding forces which can be significantly larger than originally considered - typically $10^{3}$ or more times larger in fact - and to reveal the distinct properties of the " $\alpha-G$ " coupling mechanism that could potentially be utilized in the optomechanical manipulation of matter. Exploitation of these forces could potentially be found in the helicity-dependent ${ }^{29}$ manipulation and enantiomeric separation ${ }^{30,31}$ of chiral particles, both these areas are under highly active theoretical and experimental investigations. The detailed exploration of combining these effects with discriminatory binding forces represent the scope for future work.

\section{ACKNOWLEDGMENTS}

The authors would like to thank the University of East Anglia and the Leverhulme Trust for funding this research.

\section{REFERENCES}

[1] Thirunamachandran, T., "Intermolecular interactions in the presence of an intense radiation field," Mol. Phys. 40, 393-399 (1980).

[2] Casimir, H. B. G. and Polder, D., "The influence of retardation on the London-van der Waals forces," Phys. Rev. 73, 360-372 (1948).

[3] Burns, M. M., Fournier, J.-M. and Golovchenko, J. A., “Optical binding,” Phys. Rev. Lett. 63, 1233-1236 (1989).

[4] Tatarkova, S. A., Carruthers, A. E. and Dholakia, K., "One-dimensional optically bound arrays of microscopic particles," Phys. Rev. Lett. 89, 283901 (2002). 
[5] Singer, W., Frick, M., Bernet, S. and Ritsch-Marte, M., "Self-organized array of regularly spaced microbeads in a fiber-optical trap,” J. Opt. Soc. Am. B 20, 1568-1574 (2003).

[6] Gherardi, D. M., Carruthers, A. E., Čižmár, T., Wright, E. M. and Dholakia, K., "A dual beam photonic crystal fiber trap for microscopic particles," Appl. Phys. Lett. 93, 041110 (2008).

[7] Karásek, V., Čižmár, T., Brzobohatý, O., Zemánek, P., Garcés-Chávez, V. and Dholakia, K., "Long-range onedimensional longitudinal optical binding," Phys. Rev. Lett. 101, 143601 (2008).

[8] Brzobohatý, O., Čižmár, T., Karásek, V., Šiler, M., Dholakia, K. and Zemánek, P., "Experimental and theoretical determination of optical binding forces," Opt. Express 18, 25389-25402 (2010).

[9] Demergis, V. and Florin, E.-L., "Ultrastrong optical binding of metallic nanoparticles," Nano Lett. 12, 5756-5760 (2012).

[10] Simpson, S. H., Jones, P. H., Maragò, O. M., Hanna, S. and Miles, M. J., "Optical binding of nanowires in counterpropagating beams," Proc. SPIE 8810, 881026 (2013).

[11] Yan, Z., Gray, S. K. and Scherer, N. F., "Potential energy surfaces and reaction pathways for light-mediated selforganization of metal nanoparticle clusters," Nat. Commun. 5, 3751 (2014).

[12] Čižmár, T., Dávila Romero, L. C., Dholakia, K. and Andrews, D. L., "Multiple optical trapping and binding: New routes to self-assembly," J. Phys. B: At. Mol. Opt. Phys. 43, 102001 (2010).

[13] Sukhov, S., Shalin, A., Haefner, D. and Dogariu, A., "Actio et reactio in optical binding," Opt. Express 23, 247$252(2015)$.

[14] Bradshaw, D. S. and Andrews, D. L., "Optically induced forces and torques: Interactions between nanoparticles in a laser beam," Phys. Rev. A 72, 033816 (2005).

[15] Rodríguez, J., Dávila Romero, L. C. and Andrews, D. L., "Optical binding in nanoparticle assembly: Potential energy landscapes," Phys. Rev. A 78, 043805 (2008).

[16] Dávila Romero, L. C. and Andrews, D. L., "A retarded coupling approach to intermolecular interactions," J. Phys. B: At. Mol. Opt. Phys. 42, 085403 (2009).

[17] Power, E. A. and Thirunamachandran, T., "Circular dichroism: A general theory based on quantum electrodynamics," J. Chem. Phys. 60, 3695-3701 (1974).

[18] Barron, L. D. and Buckingham, A. D., "Rayleigh and Raman scattering from optically active molecules," Mol. Phys. 20, 1111-1119 (1971).

[19] Bradshaw, D. S. and Andrews, D. L., "Chiral discrimination in optical trapping and manipulation,” New J. Phys. 16, 103021 (2014).

[20] Salam, A., "On the effect of a radiation field in modifying the intermolecular interaction between two chiral molecules," J. Chem. Phys. 124, 014302 (2006).

[21] Forbes, K. A. and Andrews, D. L., "Chiral discrimination in optical binding," Phys. Rev. A 91, 053824 (2015).

[22] Power, E. and Thirunamachandran, T., "Casimir-Polder potential as an interaction between induced dipoles," Phys. Rev. A 48, 4761 (1993).

[23] Craig, D. and Thirunamachandran, T., "New approaches to chiral discrimination in coupling between molecules," Theor. Chem. Acc. 102, 112-120 (1999).

[24] Salam, A., "Intermolecular interactions in a radiation field via the method of induced moments," Phys. Rev. A 73, 013406 (2006).

[25] Salam, A., "On the effect of a radiation field in modifying the intermolecular interaction between two chiral molecules," J. Chem. Phys. 124, 014302 (2006).

[26] Daniels, G. J., Jenkins, R. D., Bradshaw, D. S. and Andrews, D. L., "Resonance energy transfer: The unified theory revisited," J. Chem. Phys. 119, 2264-2274 (2003).

[27] Andrews, D. L. and Thirunamachandran, T., "On three-dimensional rotational averages," J. Chem. Phys. 67, 5026-5033 (1977).

[28] Andrews, D. L. and Harlow, M. J., "Phased and Boltzmann-weighted rotational averages," Phys. Rev. A 29, 2796-2806 (1984).

[29] Tkachenko, G. and Brasselet, E., "Helicity-dependent three-dimensional optical trapping of chiral microparticles," Nat. Commun. 5, 4491 (2014).

[30] Tkachenko, G. and Brasselet, E., "Optofluidic sorting of material chirality by chiral light,” Nat. Commun. 5, 3577 (2014).

[31] Bradshaw, D. S. and Andrews, D. L., "Laser optical separation of chiral molecules," Opt. Lett. 40, 677-680 (2015).

*david.andrews@physics.org 